\title{
$\mathbb{N}_{\text {Nursing Journal }}$ Global Academic
}

\section{A rastreabilidade de dispositivos médicos implantáveis e o sistema único de identificação de dispositivos: um estudo bibliométrico}

\section{The traceability of implantable medical devices and the unique device identification system: a bibliometric study}

\author{
Trazabilidad de dispositivos médicos implantables y sistema único de identificación de dispositivos: un estudio \\ bibliométrico
}

\section{Joice Alves Cabral ${ }^{1}$ \\ ORCID: 0000-0002-0411-2355 \\ Daniel Aragão Machado ${ }^{1}$ \\ ORCID: 0000-0003-0680-5291 \\ Adriana Carla Bridi \\ ORCID: 0000-0003-2018-4604 \\ Cristiano Bertolossi Marta ${ }^{2}$ \\ ORCID: 0000-00022-0635-7970 \\ Paulo Afonso Lopes da Silva ${ }^{3}$ \\ ORCID: 0000-0003-1337-2201 \\ Jorge Luiz Alves Junior ${ }^{2}$ \\ ORCID: 0000-0001-59822-6761 \\ Natalia Barroso ${ }^{4}$ \\ ORCID: 0000-0001-5781-3924 \\ Caroline de Araújo Mendes ${ }^{5}$ \\ ORCID: 0000-0002-8021-0520 \\ Valeria Mesquita Joazeiro ${ }^{6}$ \\ ORCID: 0000-0001-8976-141X \\ Luana Borges Dutra ${ }^{1}$ \\ ORCID: 0000-0002-6132-3255 \\ Raphael Barreiros Neves ${ }^{1}$ \\ ORCID: 0000-0002-0272-3187}

${ }^{1}$ Universidade Federal do Estado do Rio de Janeiro. Rio de Janeiro, Brasil.

${ }^{2}$ Universidade do Estado do Rio de Janeiro.

Rio de Janeiro, Brasil.

${ }^{3}$ Instituto Militar de Engenharia. Rio de Janeiro, Brasil.

${ }^{4}$ Instituto Nacional de Estudos e Pesquisas Educacionais Anísio Teixeira. Rio de Janeiro, Brasil.

${ }^{5}$ Prefeitura da Cidade do Rio Janeiro. Rio de Janeiro, Brasil.

6Takeda Pharmaceutical - Biolife Plasma

Center. Florida, Estados Unidos.

\section{Como citar este artigo:}

Cabral JA, Machado DA, Bridi AC, Marta CB, Silva PAL, Junior ILA, Barroso N, Mendes CA, Joazeiro VM, Dutra LB, Neves RB. A rastreabilidade de dispositivos médicos implantáveis e o sistema único de identificação de dispositivos: um estudo bibliométrico. Glob Acad Nurs. 2021;2(4):e200.

https://dx.doi.org/10.5935/2675-

5602.20200200

\section{Autor correspondente:}

Joice Alves Cabral

E-mail: joicecabral@gmail.com

Editor Chefe: Caroliny dos Santos Guimarães da Fonseca

Editor Executivo: Kátia dos Santos Armada de Oliveira

Submissão: 08-07-2021

Aprovação: 19-08-2021

\section{Resumo}

Objetivou-se sistematizar e analisar a produção científica nacional e internacional sobre a rastreabilidade de dispositivos médicos implantáveis e a utilização do Sistema Único de Identificação de Dispositivos em 126 artigos acadêmicos. Os dados foram recuperados da base de dados Scopus e analisados à luz da estatística inferencial, a fim de se chegar a conclusões sobre um grupo maior. Assim, foram adotados o coeficiente de determinação e o teste de aderência do qui-quadrado, utilizando o software Microsoft Excel ${ }^{\circledR}$ e o programa estatístico R para avaliar a atividade de produção científica relacionada com os termos rastreabilidade e identificação única do dispositivo. Foi gerado um mapa no software VOS, que proporcionou a análise dos parâmetros de comportamento e algumas apresentações dos resultados em forma de tabelas e gráficos, examinados de acordo com a estatística descritiva. Verificou-se que houve crescimento teórico e empírico no campo da rastreabilidade de dispositivos médicos implantáveis e na utilização do Sistema Único de Identificação de Dispositivos.

Descritores: Bibliometria; Programas de Rastreamento; Equipamentos e Provisões; Avaliação de Processos em Cuidados de Saúde; Monitoramento.

\section{Abstract}

The aim was to systematize and analyze the national and international scientific production on the traceability of implantable medical devices and the use of the Unique Device Identification System in 126 academic articles. Data were retrieved from the Scopus database and analyzed using inferential statistics to reach conclusions about a larger group. Thus, the coefficient of determination and the chi-square test of adherence were adopted, using the Microsoft Excel ${ }^{\circledR}$ software and the R statistical program to assess the scientific production activity related to the terms' traceability and unique identification of the device. A map was generated in the VOS software, which provided the analysis of the behavior parameters and some presentations of the results in the form of tables and graphs, examined according to descriptive statistics. It was found that there was theoretical and empirical growth in the field of traceability of implantable medical devices and in the use of the Unique Device Identification System.

Descriptors: Bibliometric; Mass Screening; Equipment and Supplies, Process Assessment, Health Care; Monitoring.

\section{Resumén}

El objetivo fue sistematizar y analizar la producción científica nacional e internacional sobre la trazabilidad de dispositivos médicos implantables y el uso del Sistema Único de Identificación de Dispositivos en 126 artículos académicos. Los datos se recuperaron de la base de datos Scopus y se analizaron utilizando estadísticas inferenciales para llegar a conclusiones sobre un grupo más grande. Así, se adoptó el coeficiente de determinación y la prueba chi-cuadrado de adherencia, utilizando el software Microsoft Excel ${ }^{\circledR}$ y el programa estadístico $\mathrm{R}$ para evaluar la actividad de producción científica relacionada con los términos trazabilidad e identificación única del dispositivo. Se generó un mapa en el software VOS, que brindó el análisis de los parámetros de comportamiento y algunas presentaciones de los resultados en forma de tablas y gráficos, examinados según estadística descriptiva. Se encontró un crecimiento teórico y empírico en el campo de la trazabilidad de dispositivos médicos implantables y en el uso del Sistema Único de Identificación de Dispositivos.

Descriptores: Bibliometría; Tamizaje Masivo; Equipos y Suministros; Evaluación de Procesos, Atención de Salud; Monitoreo. 


\section{Introdução}

Dispositivos Médicos Implantáveis (DMI) são produtos que envolvem alta tecnologia e alto valor, utilizados em procedimentos médicos, odontológicos e fisioterapêuticos, bem como no diagnóstico, tratamento, reabilitação e monitoramento de pacientes. Eles podem ser permanentes, como stents ou implantes de quadril, ou temporários, como acessos para quimioterapia ou pinos para reparar ossos fraturados, removidos após o tratamento ${ }^{1}$.

Estima-se que existam pelo menos oito mil tipos de DMI, cada grupo com características e riscos próprios associados à sua utilização. Para os fins desta pesquisa, serão considerados DMIs vinculados a um procedimento médico ou odontológico invasivo, além dos materiais utilizados como instrumentos específicos para sua implantação ${ }^{1,2}$.

Quanto aos riscos associados ao DMI, incluem-se os riscos cirúrgicos durante a sua inserção ou remoção, a possibilidade de infecção no seu local de implantação e falhas de implantação e materiais implantados ${ }^{1}$. Portanto, a segurança do paciente exige a detecção rápida de qualquer defeito - prática conhecida como vigilância de materiais, organizada a nível europeu e internacional, a fim de harmonizar as legislações sobre dispositivos médicos; isso implica na necessidade de rastrear a vida útil de um dispositivo com base no número de lote do DMI, por rastreabilidade, que se destina a identificar rapidamente os titulares de dispositivos médicos em caso de recalls de produtos, e dispositivos médicos em caso de incidente ${ }^{3}$, com cada centro responsável por organizar a rastreabilidade de seus dispositivos utilizando regras de rotulagem rígidas para garantir um uso seguro. Tal prática é um dos direitos fundamentais do paciente, uma vez que todos têm o direito de ser informados sobre seu estado de saúde.

Para realizar a rastreabilidade, foi criada uma base de dados europeia e mundial para tornar obrigatória a Identificação Única de Dispositivo (IUD) na União Europeia ${ }^{3}$, visto que a legislação francesa enumera cinco tipos de rastreabilidade: financeira, logística, jurídica, científica e relacionados à saúde.

No presente estudo, levando-se em consideração a recente abordagem científica sobre a rastreabilidade do DIM, foi difícil encontrar publicações sobre o assunto em periódicos. As publicações encontradas trataram da evolução e relevância do tema, no que se refere à segurança do paciente, UDI e vigilância pós-comercialização. Os artigos foram separados pelos idiomas inglês e francês em ordem cronológica, com auxílio da ferramenta Mendeley Desktop ${ }^{\circledR}$, para melhor organização. Vale ressaltar que não houve artigos publicados no Brasil sobre o assunto, embora a Agência Nacional de Vigilância Sanitária (Anvisa) já tenha normas quanto à rastreabilidade dos DMI.

Utilizando a modalidade de busca avançada do Portal de Periódicos da Coordenação de Aperfeiçoamento de Pessoal de Nível Superior (Capes) com as palavras-chave: rastreabilidade e dispositivo médico implantável, aplicandose os operadores booleanos AND ou OR, 36 artigos foram encontrados, 15 relacionados ao tema. No mesmo portal, ainda no modo de busca avançada, foram aplicadas as palavras-chave rastreabilidade e UDI, com AND ou OR, resultando em 127 artigos. Com filtros em política governamental, legislação de equipamentos médicos, legislação política médica, regulamentos, segurança do produto, dispositivo médico, códigos de barras, rastreabilidade, segurança do paciente, onde Foram encontrados 46 artigos relacionados a regulamentação, próteses e implantes, indústria de saúde, medicina, software, dispositivos médicos e tecnologia médica.

O artigo de 2011 Medical Devices-Balancing Regulation and Innovation de Gregory D. Curfman e Rita F. Redberg abordou o caso das próteses de quadril DePuy ${ }^{\circledR}$ metal-metal, que foram proibidas devido a sérios problemas causados a pacientes que as tinham implantado. $O$ objetivo do estudo foi discutir os sérios riscos trazidos pela entrada de dispositivos médicos não testados e potencialmente perigosos no mercado. Salienta ainda que, no mesmo ano, o Institute of Medicine divulgou relatório encomendado pela Food and Drug Administration (FDA) sobre o processo de liberação do DMI no mercado norte-americano e, entre outras recomendações, sugeria monitoramento de dispositivos médicos ao longo de seu ciclo de vida, principalmente no período pós-comercialização, por meio de um sistema formal de vigilância para $\mathrm{DMI}^{4}$.

Em vista dos eventos de recall ocorridos no mesmo ano, o artigo norte-americano 'Segurança e proteção do produto na cadeia de abastecimento global: questões, desafios e oportunidades de pesquisa' abordou os problemas de segurança que resultam na entrega de um produto inseguro ou ineficaz, por exemplo, as falsificações que podem ocorrer desde a seleção da matéria-prima até o final, chegando ao mercado por meio de um distribuidor, atacadista ou varejista. Devido às dificuldades de rastreamento da trajetória de abastecimento, principalmente ao passar por zonas de comércio internacional, a contratação é considerada um crime de difícil detecção. Nessa perspectiva, sugere-se a adoção de tecnologias de imagem e rastreamento na gestão de estoques, como códigos de barras, hologramas, reconhecimento óptico de caracteres (ROC) e identificação por radiofrequência (IPRF).

Também em 2011, o Congresso americano autorizou o FDA a estabelecer um sistema de identificação único para dispositivos médicos, que identificaria exclusivamente o produto por meio de distribuição e uso.

$\mathrm{O}$ estudo concluiu que muitos problemas nas áreas de segurança e proteção de produtos poderiam ser resolvidos pelo gerenciamento da operação. Os autores apontaram que há uma literatura cada vez maior sobre os assuntos de risco, qualidade e gestão da cadeia de suprimentos, embora tenham notado poucos trabalhos discutindo os desafios da segurança e proteção do produto. Eles também destacaram o fato de que a indústria de dispositivos médicos está trabalhando para garantir a segurança, dado o ritmo acelerado das mudanças tecnológicas, e listaram quatro áreas-chave para pesquisa em gestão de operações, relacionadas a questões de segurança e proteção: regulamentos e padrões, gestão do 
ciclo de vida do produto, rastreabilidade e gerenciamento de fornecedores e recalls ${ }^{5}$.

No artigo de revisão Vigilância e rastreabilidade de materiais, de 2016 definiu a rastreabilidade, entre outros termos relacionados ao tema, e trouxe casos de incidentes de DMI ocorridos na Europa que promoveram a reformulação do regulamento de homologação e segurança de dispositivos médicos, além de fornecer um panorama histórico da evolução da legislação da União Europeia em conjunto com a legislação dos Estados Unidos ${ }^{3}$.

Os regulamentos europeus promulgam a livre circulação de dispositivos médicos em toda a União Europeia, sendo cada Estado-Membro responsável pela segurança no seu próprio território. Isso significa que as autoridades de saúde são responsáveis por monitorar o mercado e quaisquer incidentes.

A União Europeia, em resposta ao caso de implante mamário PIP ${ }^{\circledR}$, em 2012, elaborou um novo regulamento, alterado pelo Parlamento Europeu no ano seguinte, para garantir a segurança a longo prazo das pacientes e, ao mesmo tempo, proteger a inovação. O objetivo era reforçar a avaliação da segurança e do desempenho do DMI antes da rotulagem e liberação, bem como reforçar a vigilância póscomercialização, aumentando a transparência e a coleta de dados de risco-benefício. Pela primeira vez, foi criado um sistema de controle dos órgãos regulados para o acompanhamento de suas atividades. Os dados de investigação clínica e de incidentes são recolhidos no European Databank on Medical Devices (EUDAMED), uma base de dados de acesso aberto.

A mudança na organização da rastreabilidade está em andamento e ocorrerá na forma de UDI. Essas mudanças serão refletidas em dados de etiqueta harmonizados, códigos de barras e terminologia padronizada. Segundo o estudo $^{3}$, estava prevista a criação de uma base de dados mundial até 2017, para tornar o UDI obrigatório na União Europeia; no entanto, até agora não foi estabelecido.

$O$ artigo também relata que o FDA e a União Europeia decidiram introduzir o princípio de identificação única para cada dispositivo médico e que os regulamentos para esses dispositivos foram revisados por duas diretivas europeias em 2012. O objetivo da UDI é melhorar o mercado vigilância e rastreabilidade de dispositivos médicos entre países, a fim de facilitar o acesso às bases de dados europeias da EUDAMED e da Rede Global de Sincronização de Dados (RGSD).

Mais do que facilitar a rastreabilidade, a UDI melhorará a identificação do produto, o relatório de incidentes pós-marketing e o recall do produto, além de padronizar a documentação e limitar a falsificação e o erro médico. A longo prazo, a UDI fornecerá um cartão de identificação de implante ideal, permitindo que o tipo de CMS seja recuperado junto com a identificação do paciente no banco de dados. Isso envolverá a padronização internacional de rotulagem e identificação do dispositivo médico.

O princípio UDI é baseado em três pilares: banco de dados, protocolo de nomenclatura unificada e código de barras. A base de dados EUDAMED, que entrou em vigor em maio de 2011, foi criada com o objetivo de reforçar a vigilância do mercado e a transparência em relação aos dispositivos médicos colocados no mercado europeu. Nos Estados Unidos, é o Global Unique Device Identification Database (GUDID) que envia informações ao FDA sobre os dispositivos médicos que possuem UDI. Em colaboração com a Biblioteca Nacional de Medicina dos Estados Unidos, o FDA criou um portal, denominado AccessGUDID, para disponibilizar os dados de identificação do dispositivo a todos - incluindo pacientes, cuidadores, profissionais de saúde, hospitais e indústria. Em um longo prazo, um banco de dados internacional será criado. Em relação ao segundo pilar, o protocolo para troca de dados será o Global Medical Device Nomenclature (GMDN). Esse identificador deve permitir a notificação de incidentes graves relacionados a dispositivos médicos e as medidas corretivas necessárias a serem determinadas como parte da vigilância.

O código de barras compreende dois tipos de dados: o identificador do dispositivo e o identificador de produção. O primeiro é um código numérico ou alfanumérico único que especifica o modelo ou versão do dispositivo médico, também usado como código de acesso para informações no banco de dados UDI. O segundo é um código numérico ou alfanumérico que identifica a unidade de produção do dispositivo, que compreende o número de série, o lote e o número de produção ou a data de validade. Esses dados devem ser escritos e exibidos como códigos numéricos padrão.

Na área de abrangência da União Europeia, os fabricantes devem atribuir um UDI ao dispositivo, obedecendo aos padrões internacionais, ou seja, rotular cada nível de embalagem com ele, e transmitir as informações contidas no UDI ao banco de dados EUDAMED do banco. Os importadores e distribuidores devem verificar o UDI do fabricante e inseri-lo nos dispositivos médicos junto com os dados do comprador, manipulando seus próprios sistemas de informação ${ }^{3}$.

Os importadores também devem garantir que o dispositivo foi registrado na EUDAMED. As instituições de saúde, por sua vez, precisam registrar a UDI de seus dispositivos médicos e, para determinados dispositivos, vinculá-la ao paciente em questão. Ambos devem garantir os meios de leitura dos rótulos e a comunicação com a base de dados internacional. Nos Estados Unidos, a meta era estabelecer o sistema UDI até 2015; e, na Europa, o UDI se tornaria obrigatório para todos os dispositivos médicos em 2017 [3]; atualmente, essas metas permanecem em fase de estruturação.

Atualmente, a vigilância de materiais encontra-se perfeitamente estruturada a nível nacional nos EstadosMembros da União Europeia, contando com o envolvimento ativo dos profissionais de saúde. Assim, pode-se dizer que os novos regulamentos da União Europeia melhoraram a segurança, a inovação e a marcação de conformidade europeia (CE) dos dispositivos médicos. Com a implantação da UDI e das bases de dados europeias e internacionais, prevista para 2016, segundo o autor, a segurança da circulação de dispositivos médicos em todo o mundo será 
A rastreabilidade de dispositivos médicos implantáveis e o sistema único de identificação de dispositivos: um estudo bibliométrico Cabral JA, Machado DA, Bridi AC, Marta CB, Silva PAL, Junior JLA, Barroso N, Mendes CA, Joazeiro VM, Dutra LB, Neves RB

maximizada, otimizando também o manejo do paciente em caso de problemas ${ }^{3}$.

No Brasil, o controle sanitário de dispositivos médicos é o mesmo adotado na maioria dos países onde existe infraestrutura e força legal para atuar neste segmento, como no Canadá e nos Estados Unidos, bem como segue o modelo recomendado pela Organização Panamericana de Saúde (OPAS) e Organização Mundial da Saúde (OMS). Esse controle abrange dois fatores de risco principais: o produto e o uso. No primeiro caso, o controle está relacionado ao registro do produto e o uso. No primeiro caso, o controle está relacionado ao registro do produto e ao conhecimento das condições de fabricação; portanto, para a fase de pré-comercialização; quanto ao uso, o controle concentra-se nos problemas associados ao uso do produto na fase de pós-comercialização, sendo também conhecido como tecno vigilância ${ }^{1,2}$.

Aliada à regularidade sanitária dos dispositivos implantáveis, faz-se necessário observar as questões relacionadas à rastreabilidade de produtos médicos, conforme previsto na Resolução n. 0 2, de 25 de janeiro de 2010, feito pela Anvisa, da qual são extraídos dados que auxiliam na o processo de investigação, uma vez que, em inúmeras situações, as investigações de surtos infecciosos envolvendo implantáveis esbarram na falta de registros de rastreabilidade, por vezes tornando os casos inconclusivos ${ }^{1,6}$.

No âmbito da segurança do paciente, propõe-se que a equipe envolvida no procedimento registre todos os atributos do DIM a ser implantado no paciente durante o ato cirúrgico na descrição cirúrgica, na ficha de consumo da sala e no próprio paciente. registro. Ou seja: nome do material, fabricante ou importador, marca e modelo, tamanho, lote, registro na Anvisa, validade, data de uso, nome do profissional responsável pelo procedimento, paciente, fornecedor, número da fatura e descrição do implante realizada. Além disso, as ocorrências também devem ser registradas detalhadamente, como eventos adversos, não conformidades apresentadas pelo CMS ou qualquer outro desvio de qualidade ${ }^{7}$.

Desse modo, a criação e padronização da UDI no âmbito das agências reguladoras internacionais, incluindo a Anvisa, visa estabelecer um sistema único e globalmente harmonizado, capaz de identificar produtos médicos. Com este sistema, profissionais de saúde e pacientes não precisarão mais acessar fontes múltiplas, inconsistentes e incompletas na tentativa de identificar um dispositivo médico e seus principais atributos. É importante observar que os benefícios da UDI estão diretamente ligados à capacidade de rastrear todo e qualquer produto para saúde, especialmente o DMI, em toda a cadeia de produção ${ }^{8}$.

Este trabalho é um estudo bibliométrico que visa; identificar os periódicos mais dedicados e; identificar as palavras mais utilizadas que melhor descrevem o tema e determinar o grupo de elite de autores ${ }^{9-12}$.

\section{Metodologia}

Trata-se de um estudo bibliométrico realizado exclusivamente com dados secundários de domínio público; por esse motivo, não foi necessária a aprovação do Comitê de Ética em Pesquisa, conforme recomendações da Resolução n.o 466, do Conselho Nacional de Saúde ${ }^{13}$.

Para avaliar os indicadores de relevância, as principais leis bibliométricas utilizadas são a de Lotka, de Bradford, de Zipf e a Teoria de Goffman, que enfocam, respectivamente, a produtividade de periódicos e autores, a frequência de ocorrência de palavras, a estimativa do coeficiente de crescimento e decaimento de uma área disciplinar, bem como a relevância de linhas de pesquisa em áreas específicas do conhecimento $9,10,12,14,15$.

Para a obtenção dos dados bibliométricos, foi utilizada a base de dados Scopus $^{\circledR}$, que possui ferramentas avançadas como seus filtros de busca, proporcionando mais qualidade à busca. Os termos selecionados em inglês foram "Unique Device Identification", com a string ALL ("Unique Device Identification") AND (excluir (subjarea, "bioc") OR excluir (subjarea, "phar") OR exclude (subjarea, "ceng") AND (excluir (subjarea, "math") OR excluir (subjarea, "phys") AND (excluir (subjarea, "chem") AND excluir (subjarea, "neur"), visando uma gama maior de trabalhos relacionados ao IMD rastreabilidade, vale ressaltar que os dados que não são abertos são interpretados pelo Scopus ${ }^{\circledR}$ como indefinidos, enquanto outros são classificados como diferentes, como o número total de artigos.

Figura 1. Fluxograma para obtenção dos dados da pesquisa. Rio de Janeiro, RJ, Brasil, 2019

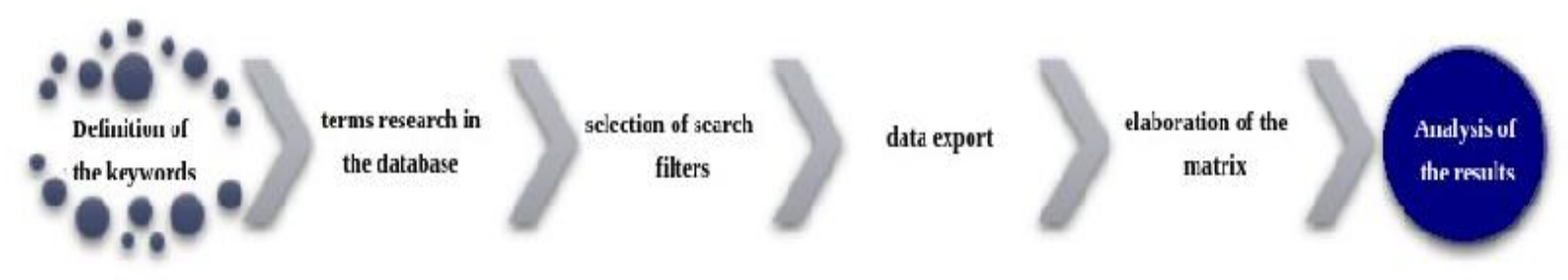


A rastreabilidade de dispositivos médicos implantáveis e o sistema único de identificação de dispositivos: um estudo bibliométrico Cabral JA, Machado DA, Bridi AC, Marta CB, Silva PAL, Junior JLA, Barroso N, Mendes CA, Joazeiro VM, Dutra LB, Neves RB

Após esse primeiro processo, filtros de refinamento de pesquisa foram adicionados com os seguintes parâmetros: todos os períodos, países, idiomas, área do conhecimento (ciências da saúde, medicina, engenharia, enfermagem, ciência da computação, ciência dos materiais, ciências sociais, ciência da decisão, negócios e gestão) e tipos de documentos.
Por fim, para avaliar a produção científica sobre o termo "Unique Device Identification", foram aplicadas as leis de Lotka [9], Bradford [10], Zipf [11-12] e Price [16-17], bem como a co-citação. Por meio do software $\operatorname{VOS}^{\circledR}$, foram gerados mapas e, a partir deles, analisados os parâmetros de comportamento. Os resultados apresentados na forma de Quadros e Gráficos foram examinados à luz da estatística descritiva, conforme apresentado no Quadro 1.

Quadro 1. Leis e princípios da bibliometria. Rio de Janeiro, RJ, Brasil, 2019

\begin{tabular}{|c|c|c|}
\hline \multicolumn{3}{|c|}{ Bibliometria } \\
\hline Leis e princípios & Título dos periódicos & Principais Aplicações \\
\hline Bradford's & Foco de estudo & $\begin{array}{l}\text { Estimativa de graus de relevância de } \\
\text { títulos de periódicos em áreas do } \\
\text { conhecimento. }\end{array}$ \\
\hline Lotka's & Autores & $\begin{array}{l}\text { Estimativa dos graus relativos de } \\
\text { relevância dos autores nas áreas. }\end{array}$ \\
\hline Zipf's & Words & $\begin{array}{l}\text { Análise conceitual da redação científica } \\
\text { e indexação automática ou } \\
\text { semiautomática de artigos científicos. }\end{array}$ \\
\hline Co-citações & Citações & $\begin{array}{c}\text { Estimativa dos graus relativos de } \\
\text { conexão de dois ou mais artigos (análise } \\
\text { prospectiva). }\end{array}$ \\
\hline Teoria das epidemias de Goffman & Citações & $\begin{array}{l}\text { Estimativa do grau de crescimento e } \\
\text { declínio de uma área disciplinar e da } \\
\text { importância das linhas de pesquisa em } \\
\text { áreas específicas do conhecimento. }\end{array}$ \\
\hline Lei do elitismo (Lei de Prince) & Citações & $\begin{array}{l}\text { Identificação e descrição da elite, } \\
\text { formada por autores que participaram } \\
\text { intensamente da produção científica } \\
\text { em áreas específicas do conhecimento. }\end{array}$ \\
\hline
\end{tabular}

\section{Resultados}

Devido à grande quantidade de dados, a discussão foi dividida em subtópicos.

\section{Distribuição de artigos por países}

Por meio dos dados da pesquisa, foram obtidas informações dos países que publicaram sobre o tema deste artigo. Conforme demonstrado na Tabela 1, os Estados Unidos apresentam a maior produtividade relacionada à UDI, com 74 artigos publicados (49\%), enquanto no Brasil nenhuma publicação foi encontrada.

\section{Distribuição de artigos por idioma}

Com os dados da pesquisa, os idiomas que publicaram sobre o tema em estudo emergiram, que o maior número de artigos publicados foi em inglês, totalizando 119 (92\%).

\section{Distribuição de artigos por ano}

A Figura 2 mostra que o número de publicações sobre o tema aumentou, com pico no número de publicações em 2014, com 28 artigos (22\%).

Tabela 1. Distribuição dos artigos por países. Rio de Janeiro, RJ, Brasil, 2019

\begin{tabular}{|c|c|c|}
\hline Países & Número de Artigos & $\%$ \\
\hline Estados Unidos & 74 & $49 \%$ \\
\hline Reino Unido & 10 & $7 \%$ \\
\hline França & 9 & $6 \%$ \\
\hline Alemanha & 7 & $5 \%$ \\
\hline Australia & 5 & $3 \%$ \\
\hline Canada & 4 & $3 \%$ \\
\hline Finlandia & 3 & $2 \%$ \\
\hline India & 3 & $2 \%$ \\
\hline Italia & 3 & $2 \%$ \\
\hline Suécia & 3 & $2 \%$ \\
\hline Suíça & 2 & $1 \%$ \\
\hline Turquia & 2 & $1 \%$ \\
\hline Austria & 1 & $1 \%$ \\
\hline Belgica & 1 & $1 \%$ \\
\hline China & 1 & $1 \%$ \\
\hline Cuba & 1 & $1 \%$ \\
\hline Republica Checa & 1 & $1 \%$ \\
\hline
\end{tabular}


A rastreabilidade de dispositivos médicos implantáveis e o sistema único de identificação de dispositivos: um estudo bibliométrico Cabral JA, Machado DA, Bridi AC, Marta CB, Silva PAL, Junior JLA, Barroso N, Mendes CA, Joazeiro VM, Dutra LB, Neves RB

\begin{tabular}{|c|c|c|}
\hline Japão & 1 & $1 \%$ \\
\hline México & 1 & $1 \%$ \\
\hline Polônia & 1 & $1 \%$ \\
\hline Coréia do Sul & 1 & $1 \%$ \\
\hline Indefinido & 18 & $12 \%$ \\
\hline Total* & 152 & $100 \%$ \\
\hline
\end{tabular}

Nota: * Desse total, apenas 126 possuem dados abertos.

Figura 2. Distribuição de artigos por ano 2006-2018. Rio de Janeiro, RJ, Brasil, 2019.

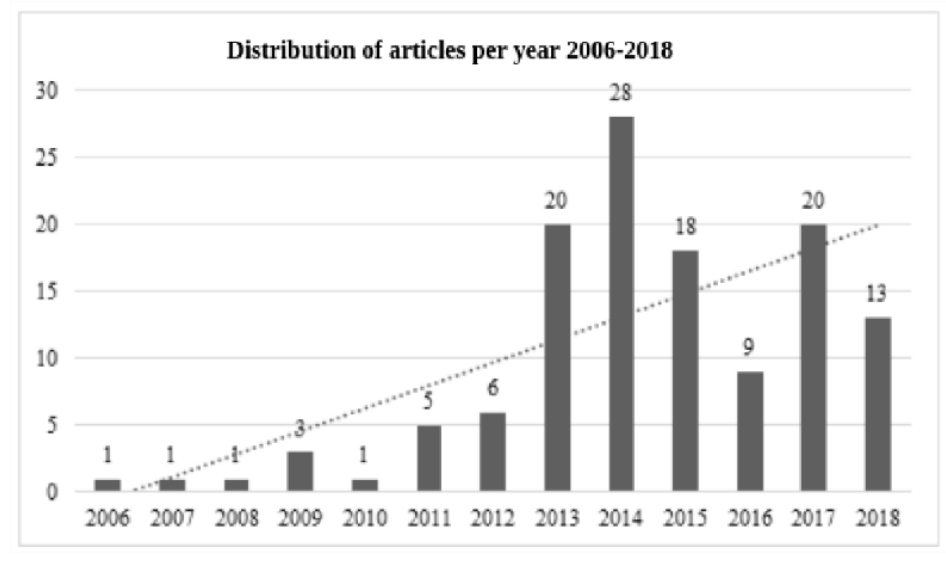

\section{Distribuição de artigos por autor: Lei de Price}

De acordo com a Tabela 2, de um total de 126 artigos e 96 autores, 62 artigos (49\%) foram produzidos por 13 autores (14\%), o que dá uma média de 4,77 artigos por autor, o que corrobora a Lei de Price, visto que houve uma elite de autores mais produtivos, uma vez que a raiz quadrada do número total de autores representa, pelo menos, metade da produção dos artigos produzidos

\begin{tabular}{lccc}
\multicolumn{2}{c}{ Tabela 2. Porcentagem acumulada de produção por número de autores. Rio de Janeiro, RJ, Brasil, 2019 } \\
\hline Número de autores & Número de artigos & Acumulativo \% \\
\hline 1 & & & $27,0 \%$ \\
2 & 34 & $27,0 \%$ & $40,5 \%$ \\
3 & 17 & $13,5 \%$ & $54,8 \%$ \\
4 & 18 & $14,3 \%$ & $68,3 \%$ \\
5 & 17 & $13,5 \%$ & $78,6 \%$ \\
6 & 13 & $10,3 \%$ & $84,9 \%$ \\
7 & 8 & $6,3 \%$ & $87,3 \%$ \\
8 & 3 & $2,4 \%$ & $88,1 \%$ \\
9 & 1 & $0,8 \%$ & $92,9 \%$ \\
10 & 6 & $4,8 \%$ & $95,2 \%$ \\
13 & 3 & $2,4 \%$ & $97,6 \%$ \\
16 & 3 & $2,4 \%$ & $98,4 \%$ \\
Total & 1 & $0,8 \%$ & $100,0 \%$ \\
\hline
\end{tabular}

O mapa feito no VOSviewer ${ }^{\circledR}$ corrobora os gráficos produzidos com base nos dados obtidos no Scopus. No que diz respeito à co-citação e autoria das publicações, Marinac-
Dabić, JS Ross e A Sedrakyan se destacaram entre os 10 autores mais produtivos Figura 3.

Figura 3. Mapa de co-autoria. Rio de Janeiro, RJ, Brasil, 2019

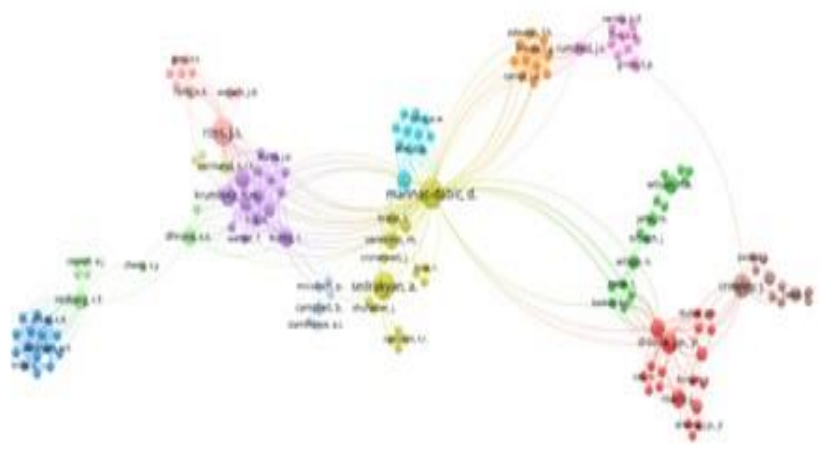




\section{Aplicação da Lei de Bradford}

De acordo com a Lei de Bradford, se os periódicos são organizados em ordem decrescente de produtividade em um determinado tópico, eles podem ser distribuídos em um grupo de periódicos mais especificamente dedicados a esse assunto e em diferentes grupos ou zonas contendo o mesmo número de artigos que o grupo, sempre que o número de periódicos e zonas sucessivas for igual a 1: $n: n 2$, considerando $n=3$ e a frequência de 1: 3: 9. Como neste estudo existem 88 periódicos e o núcleo contém uma parte deles, zona I terá três partes; e zona II, nove partes.

Assim, 88 foi dividido pelo número total de peças; neste caso, $13(1+3+9)$. Portanto, 88/13 = 6,77, ou seja, foram obtidos aproximadamente 7 (Tabelas 3 e 4). Portanto, a frequência esperada para o núcleo é 7 , para a zona I é 20 e para a zona II é 61.

Tabela 3. Número esperado de periódicos versus número obtido na amostra. Rio de Janeiro, RJ, Brasil, 2019

\begin{tabular}{lcc}
\multicolumn{2}{c}{ Tabela 3. Número esperado de periódicos versus número obtido na amostra. Rio de Janeiro, RJ, Brasil, 2019} \\
\hline Zonas & Número de periódicos esperados & Numero de revistas obtidas \\
\hline Core & 7 & 11 \\
Zona I & 20 & 35 \\
Zona II & 61 & 42 \\
\hline
\end{tabular}

Tabela 4. Número obtido na amostra. Rio de Janeiro, RJ, Brasil, 2019

\begin{tabular}{lcccc}
\hline Zonas & Número de periódicos & $\begin{array}{c}\text { Multiplicador de } \\
\text { Bradford }\end{array}$ & Artigos Acumulados & Rank \\
\hline Core & 11 & & 42 & 11 \\
Zona I & 35 & 3,181818182 & 84 & 46 \\
Zona II & 42 & 1,2 & 126 & 88 \\
\hline
\end{tabular}

O teste de Qui-quadrado de aderência com nível de significância de $5 \%$ foi realizado para verificar se esses dados obedeciam à Lei de Bradford. Com o valor de $p<0,05$, há evidências significativas de que o número de periódicos na UDI não segue essa lei.

\section{Jornal SCImago Rank boxplot}

Neste estudo, três caixas foram colocadas lado a lado, a fim de comparar a variabilidade do SCImago Journal Rank (SJR) entre as três zonas de acordo com a Lei de Bradford. Com este tipo de gráfico, obteve-se a mediana, o primeiro quartil, o terceiro quartil, o menor valor e o maior valor do conjunto de dados, bem como os outliers.

Quanto aos quartis, percebeu-se que existe uma distribuição simétrica entre as zonas II e III quanto à variação dos valores do SJR, uma vez que a mediana fica no centro de cada retângulo. No entanto, na zona III, um valor SJR mais alto foi alcançado do que na zona II. Na zona I, por outro lado, encontram-se os maiores valores do SJR, o que configura um prestígio comparativamente maior entre os periódicos da zona I, ao contrário das zonas II e III, conforme mostra a Figura 4.

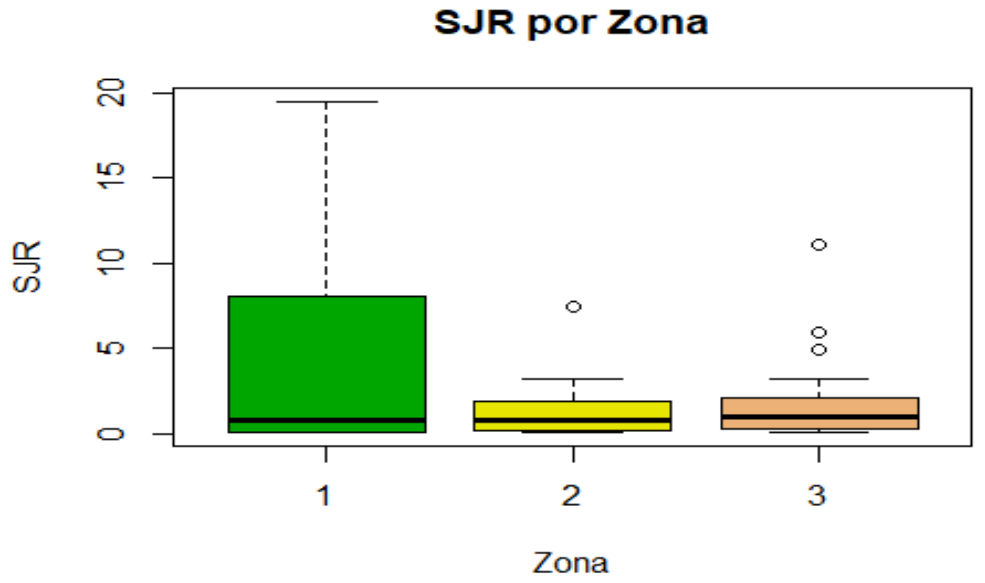

\section{Primeira lei de Zipf}

Um dos dados gerados pela base de dados Scopus ${ }^{\circledR}$ foi a lista de palavras utilizadas pelos autores nos artigos desta pesquisa. A partir dela, aplicamos a Primeira Lei Zipf como forma de inferir sobre os principais assuntos tratados nos artigos selecionados com as palavras a eles associadas. Os termos utilizados são de origem inglesa, considerando que o conjunto de dados recuperado do banco de dados utilizava apenas esse idioma.

Dessa forma, palavras que tinham o mesmo significado ou se repetiam no plural e no singular foram unificadas, de modo que não houve repetição de termos que comprometessem uma análise posterior. Após esse procedimento de filtragem, 830 palavras únicas e exclusivas 
A rastreabilidade de dispositivos médicos implantáveis e o sistema único de identificação de dispositivos: um estudo bibliométrico Cabral JA, Machado DA, Bridi AC, Marta CB, Silva PAL, Junior JLA, Barroso N, Mendes CA, Joazeiro VM, Dutra LB, Neves RB foram mapeadas e, a seguir, promovida a tabulação correspondente. Vale ressaltar que os artigos apresentaram 830 palavras, com diferentes níveis de frequência. Para o Tabela 5, na qual a série de palavras foi ordenada de acordo com sua frequência no conjunto de dados recuperado da cálculo da equação da Primeira Lei de Zipf, foi elaborada a base Scopus.

Tabela 5. Frequência de palavras para aplicação da primeira Lei de Zipf. Rio de Janeiro, RJ, Brasil, 2019

\begin{tabular}{|c|c|c|c|}
\hline $\begin{array}{c}\text { Número de palavras } \\
\text { classificadas em "R" } \\
\text { ordenado }\end{array}$ & "R" Ordenado & $\begin{array}{c}\text { Ocorrência e } \\
\text { frequência "F" }\end{array}$ & Constante da Primeira Lei de Zipf (R. F = C) \\
\hline 1 & 1 & 125 & 125 \\
\hline 1 & 2 & 92 & 184 \\
\hline 1 & 3 & 73 & 219 \\
\hline 1 & 4 & 69 & 276 \\
\hline 1 & 5 & 59 & 295 \\
\hline 1 & 6 & 58 & 348 \\
\hline 1 & 7 & 56 & 392 \\
\hline 1 & 8 & 47 & 376 \\
\hline 1 & 9 & 45 & 405 \\
\hline 1 & 10 & 41 & 410 \\
\hline 1 & 11 & 38 & 418 \\
\hline 1 & 12 & 34 & 408 \\
\hline 3 & 13 & 31 & 403 \\
\hline 1 & 14 & 28 & 392 \\
\hline 1 & 15 & 25 & 375 \\
\hline 1 & 16 & 24 & 384 \\
\hline 1 & 17 & 23 & 391 \\
\hline 2 & 18 & 22 & 396 \\
\hline 2 & 19 & 21 & 399 \\
\hline 1 & 20 & 19 & 380 \\
\hline 2 & 21 & 18 & 378 \\
\hline 1 & 22 & 14 & 308 \\
\hline 5 & 23 & 13 & 299 \\
\hline 3 & 24 & 12 & 288 \\
\hline 7 & 25 & 11 & 275 \\
\hline 6 & 26 & 10 & 260 \\
\hline 6 & 27 & 9 & 243 \\
\hline 16 & 28 & 8 & 224 \\
\hline 12 & 29 & 7 & 203 \\
\hline 6 & 30 & 6 & 180 \\
\hline 19 & 31 & 5 & 155 \\
\hline 37 & 32 & 4 & 128 \\
\hline 53 & 33 & 3 & 99 \\
\hline 104 & 34 & 2 & 68 \\
\hline 529 & 35 & 1 & 35 \\
\hline
\end{tabular}

Os resultados dos testes da Primeira Lei de Zipf podem ser melhor visualizados nas Figuras 5 e 6 , elaboradas com o objetivo de ilustrar o comportamento da constante $C$ de Zipf ao longo das ordens seriais das palavras. Quanto à Figura 5, era esperada uma parábola com linha de tendência constante, para que a Primeira Lei de Zipf pudesse ser aplicada à produção científica sobre IDs, ou seja, a linha tracejada não demonstra a tendência de constância, sinalizada por Zipf, quando o a ordem em série é multiplicada pela frequência de ocorrência. Podemos observar que o coeficiente de determinação $\left(R^{2}\right)$ é igual a $93,35 \%$, o que se ajusta bem ao modelo aplicado. Porém, a constante $C$ da Lei de Zipf não segue o que era esperado. Tendo em vista que existe uma dispersão heterogênea e que essa lei se aplica a palavras de alta frequência, podemos assumir que esse é o motivo da não aplicação da primeira lei, onde $C=R \times F$ (Figura 5). Na Figura 6, o coeficiente de determinação $\left(R^{2}\right)$ é igual a $97,46 \%$, o que se ajusta bem ao modelo aplicado. Observamos o comportamento das palavras, no que diz respeito à ordem das séries e à 
A rastreabilidade de dispositivos médicos implantáveis e o sistema único de identificação de dispositivos: um estudo bibliométrico Cabral JA, Machado DA, Bridi AC, Marta CB, Silva PAL, Junior JLA, Barroso N, Mendes CA, Joazeiro VM, Dutra LB, Neves RB frequência de ocorrência: quanto maior a ordem das séries, menor a frequência com que as palavras ocorrem, corroborando com o que Zipf recomendou. Na Figura 5, é possível perceber que a linha de tendência se aproxima da linha de função do gráfico.

Figura 5. Dispersão - Constante da Primeira Lei de Zipf (R. F = C). Rio de Janeiro, RJ, Brasil, 2019

\section{Gráfico de dispersão: Constante da Primeira Lei de Zipf $(r$. f = c)}

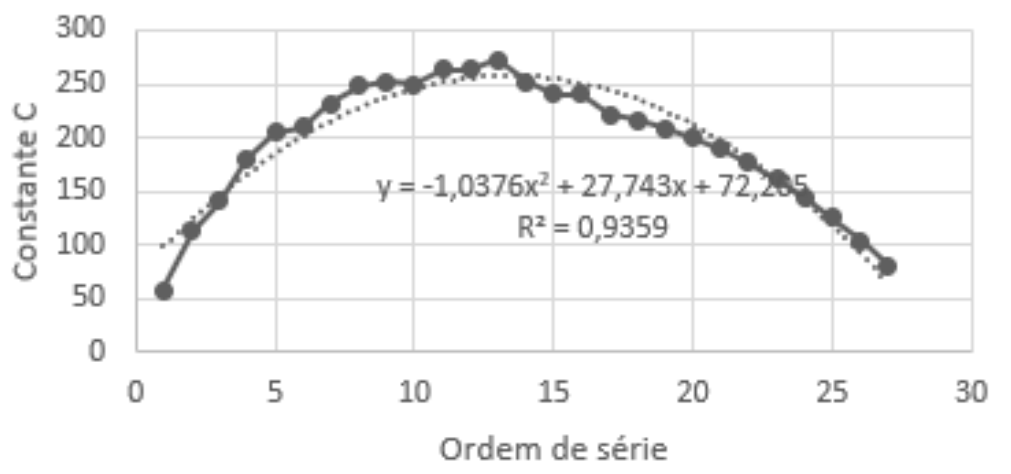

Figura 6. Dispersão - Freqüência de ocorrência ordem das séries "F” versus "R". Rio de Janeiro, RJ, Brasil, 2019

\section{Gráfico de dispersão: Frequência de ocorrência "F" versus ordem de série " $R$ "}

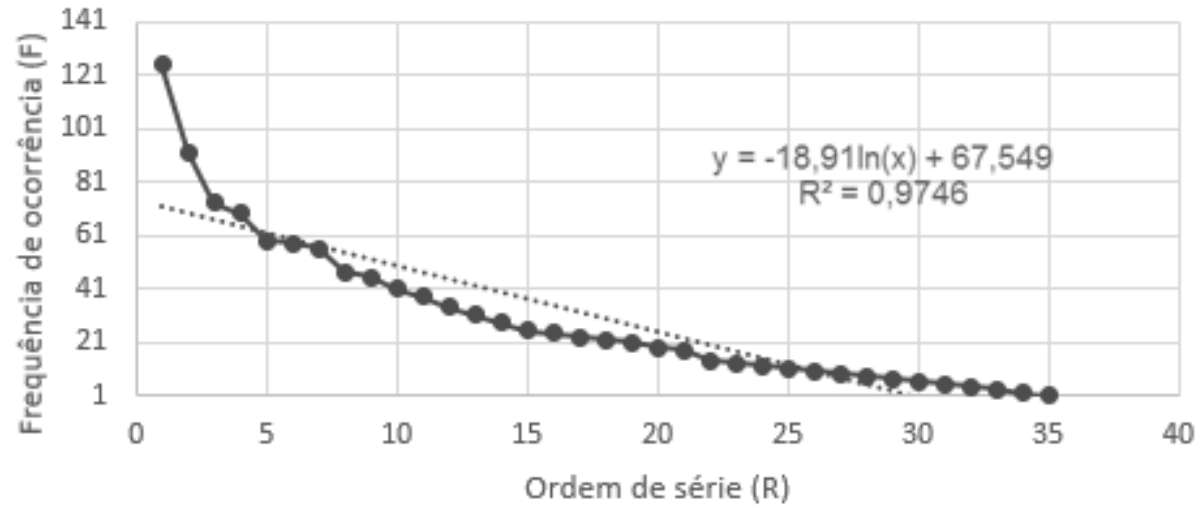

Segunda Lei de Zipf da Lei de Zipf-Booth: Ponto de Transição de Goffman (T)

Neste trabalho, o ponto $T$ de Goffman está localizado ao redor das palavras com frequência 65 , correspondendo ao conjunto: vigilância pós-mercado / vigilância pós-comercialização de produtos / vigilância póscomercialização / vigilância de produtos / vigilância de produtos, pós-comercialização / pós-comercialização / póscomercialização e dispositivos médicos / dispositivos médicos.

$$
\begin{gathered}
529 \times 8=4.232 \\
4.232-1=4.231 \\
\sqrt{4231}=65,04
\end{gathered}
$$

\section{Esferas Zipf}

Além de aplicar a fórmula da Primeira Lei de Zipf, neste estudo os dados foram tratados nas esferas ou zonas da Lei de Zipf, tendo como primeira esfera as palavras mais comuns dentro do texto e na segunda esfera palavras que ocorreram em um número menor do que os mencionados na primeira esfera. Como não são de uso comum, são considerados mais importantes nas publicações relacionadas ao UDI. Na terceira esfera estão as palavras consideradas ruídos no texto.

Com base na matemática, é possível traçar uma série de fenômenos apenas analisando a frequência com que certas palavras aparecem em um texto. A base matemática da lei é:

$$
\begin{aligned}
& \checkmark T W=F S \\
& \checkmark F S=S S
\end{aligned}
$$

Onde: "TW" é o total de palavras (ocorrências); "FS" é a primeira esfera; "SS" é a segunda esfera.

Este estudo tem: $\vee 830=28,80$ (primeira esfera), $\vee$ $28,80=5,36$ (segunda esfera). 
A rastreabilidade de dispositivos médicos implantáveis e o sistema único de identificação de dispositivos: um estudo bibliométrico Cabral JA, Machado DA, Bridi AC, Marta CB, Silva PAL, Junior JLA, Barroso N, Mendes CA, Joazeiro VM, Dutra LB, Neves RB

Assim, a primeira esfera representa as palavras que possuem pelo menos 29 ocorrências, e a segunda esfera é composta por aquelas que possuem pelo menos cinco ocorrências (Tabela S1 e Tabela S2).

\section{Produtividade por área de conhecimento: Lei de Lotka}

No que se refere à área de conhecimento relacionada ao tema desta pesquisa, dos 126 artigos, a área da medicina publicou $60 \%$ dos artigos encontrados; engenharia, $17 \%$; e enfermagem, $3 \%$.

\section{Tipo de documento}

Os dados obtidos nesta pesquisa revelaram os tipos de publicação. A fim de solucionar eventuais erros de tradução quanto aos tipos de documentos, optou-se por manter os tipos de documentos em inglês. Assim, constatouse que, do total de publicações recuperadas na base de dados, o maior percentual foram Artigos, com 57\%.

\section{Produtividade por Afiliação}

Dos dados da pesquisa, surgiram 160 entidades cujas publicações estão vinculadas a elas. Optamos por destacar, na Tabela 7, as 10 entidades que mais publicaram, o que atinge, juntas, um total de 79 publicações. O FDA obteve $18(6 \%)$ artigos a ela afiliados e, conforme mostra a Tabela 6, as instituições apresentadas são dos Estados Unidos, o que confirma o maior percentual de publicações apresentadas neste estudo. O percentual das demais instituições apresentou representatividade inferior a $1 \%$ no total de publicações.

Tabela 6. Produtividade por afiliação: as 10 entidades mais publicadas. Rio de Janeiro, RJ, Brasil, 2019

\begin{tabular}{lc}
\hline Instituições & $\begin{array}{c}\text { Número de } \\
\text { artigos }\end{array}$ \\
\hline Food and Drug Administration & 18 \\
Harvard Medical School & 13 \\
Food and Drug Administration, Center for Devices and Radiological Health & $4 \%$ \\
Weill Cornell Medical College & $3 \%$ \\
Yale University School of Medicine & $2 \%$ \\
Beth Israel Deaconess Medical Center & 7 \\
Yale University & $6 \%$ \\
Yale-New Haven Hospital & 6 \\
UCL & $2 \%$ \\
Duke Clinical Research Institute & $2 \%$ \\
\hline
\end{tabular}

\section{Discussão}

O estudo bibliométrico compreende técnicas de leitura, seleção, arquivamento e arquivamento de temas de interesse para a pesquisa, com o objetivo de conhecer as contribuições científicas feitas sobre determinado assunto. Com o objetivo de retratar o comportamento e o desenvolvimento da produção científica em uma determinada área do conhecimento, dentro de uma abordagem quantitativa ${ }^{16}$.

O assunto do DMI é complexo, pois envolve múltiplos atores e interesses inter-relacionados: pacientes, médicos, outros profissionais de saúde, fabricantes, fornecedores de insumos e prestadores de serviços de saúde, cada um assumindo sua cota de responsabilidade na cadeia de uso.

No Brasil, o Ministério da Saúde elaborou o Manual de Boas Práticas de Gestão de Órteses, Próteses e Materiais Especiais, e a Anvisa possui diretrizes para regulamentar o mercado interno e o uso de produtos para saúde, bem como monitorar o uso de DMI ${ }^{7}$.

Dada a relevância do tema (ID), vinculada ao IDM e à rastreabilidade, o número de publicações encontradas foi pequeno - 126 artigos, se comparado a outros temas relacionados à segurança do paciente, por exemplo.
A Lei de Lotka está relacionada ao cálculo da produtividade dos autores de artigos científicos. De acordo com essa lei, existe a coexistência, no âmbito da especialidade científica, de um pequeno número de autores que apresentam alto grau de produtividade, em confronto com muitos cientistas menos produtivos. Portanto, verificase que alguns pesquisadores, teoricamente com maior autoridade em uma área do conhecimento, produzem muito, enquanto outros pesquisadores, teoricamente com menor influência, produzem pouco ${ }^{14,15}$.

Considerando a metodologia utilizada neste estudo, por meio dos dados recuperados do banco de dados, constatou-se que os Estados Unidos apresentam a maior produtividade no tema, com $49 \%$ dos artigos publicados. Quanto ao idioma das publicações, a maioria dos artigos foi publicada na língua inglesa (92\%). Ressalta-se que, apesar da relevância mundial da língua inglesa, outros países, como a França, publicaram artigos detalhados sobre o tema da rastreabilidade do IDM.

No que se refere à distribuição dos artigos por ano, constatou-se que, no período de 2006 a 2018, houve um aumento no número de publicações, contrariando a tendência de declínio das publicações. Observou-se também que em 2014 houve um pico de publicações, representando $22 \%$ dos artigos publicados. Portanto, a Lei de Price diz que 
o número de membros da elite corresponde à raiz quadrada do número total de autores, e metade da produção total é tomada como critério para saber se a elite é produtiva ou não. Quanto à Lei de Price, $49 \%$ das publicações foram produzidas por 13 autores, sendo estes os mais produtivos no tema deste estudo ${ }^{14,16,17}$.

Seguindo a Lei de Bradford, os testes mostraram que o comportamento deveria seguir a razão de 7:20:61 (frequência esperada), e a razão observada foi de 11:35:42. Considerando a significância de $5 \%$ para o teste Quiquadrado, obteve-se valor de $p<0,05$, comprovando que os achados deste estudo não seguem a Lei Bibliométrica de Bradford $^{2,10}$.

Quanto às palavras, todas na língua inglesa, foi aplicada a Primeira Lei de Zipf, mostrando grande dispersão e heterogeneidade entre elas, não encontrando uma constante de acordo com a lei. Esse achado surpreendeu os pesquisadores do presente estudo.

Referindo-se à Segunda Lei de Zipf e ao ponto T de Goffman, foram encontradas palavras que se relacionam com o tema deste estudo: "vigilância pós-mercado" e "dispositivos médicos". A vigilância pós-comercialização associada a dispositivos médicos está no cerne do uso de UDI, uma vez que integrar a identificação de DMI facilita sua rastreabilidade, alcançando melhor vigilância e regulamentação.

Analisando a teoria da Segunda Lei de Zipf, palavras com baixa frequência tiveram a mesma ocorrência daquelas com alta frequência. Por esse motivo, a Segunda Lei de Zipf, modificada por Booth, apresenta o comportamento das palavras de baixa frequência de ocorrência. Nessa região, nota-se que existem muitas palavras com a mesma frequência ${ }^{14,19}$.

Dada a importância do tema UDI e da rastreabilidade do $\mathrm{DMI}$, identificou-se a necessidade de inserir, na discussão dos dados, outro paralelo que poderia ser traçado neste artigo: a relevância dos estudos realizados em outros países, confirmando a necessidade de estimular a publicação sobre o tema, bem como a unificação mundial no processo de rastreabilidade do $\mathrm{DMI}$, tendo em vista os casos de falhas de processo, desde a fabricação até o pósmarketing, além do desconhecimento do assunto por parte dos os autores envolvidos: fabricantes, fornecedores, hospitais, equipes de saúde e pacientes.

O funcionamento adequado do dispositivo médico e o bem-estar de seus destinatários deveriam ter maior prioridade para o FDA e os fabricantes de DMI, mas foi apontada ao Congresso dos Estados Unidos a necessidade de adotar novas ações a fim de proteger os pacientes com material implantável. No estudo foi relatado que os pacientes com DMRI revogada não tinham direito a uma visita ao médico, sem nenhum custo para eles, para discutir as implicações de saúde ou recall20.

Um dos estudos reforçou que a regulação do risco de dispositivos médicos é uma área negligenciada nas pesquisas das ciências sociais e políticas de saúde, principalmente no que diz respeito à utilidade da rastreabilidade, tão necessária para que as informações de um recall cheguem ao paciente ou ao médico. de forma padronizada, por meio de um único cadastro. Ele também relatou que a ideia de prontuários e bancos de dados de pacientes era, na época, ser considerada pelos Estados Unidos e pela União Europeia, e que ambos estavam em processo de introdução da UDI ${ }^{21}$.

Outro estudo buscou identificar as "melhores práticas" entre esses países, que poderiam apoiar as metas de saúde pública de todos os sistemas regulatórios do DMS. Embora apenas medidas quantitativas limitadas de vigilância pós-mercado guiem as decisões de políticas de saúde, o estudo avaliou a variedade de estratégias de vigilância pósmercado em quatro grandes mercados de dispositivos médicos: Estados Unidos, União Europeia, Japão e China ${ }^{22}$.

Para os autores de um dos artigos analisados, considerando que um número crescente de dispositivos têm sido aplicados e utilizados na assistência médica nos últimos anos - a cada ano, mais de 300.000 articulações artificiais de quadril e joelho são implantadas somente na Alemanha - e levando em consideração que mais tecnologias de informação e comunicação serão utilizadas na área da saúde, resultando em um número crescente de registros clínicos, estudos de coorte, espera-se que os dados relacionados aos dispositivos médicos venham cada vez mais do campo da pesquisa em serviços de saúde, e isso, por sua vez, se tornará uma fonte cada vez mais importante de conhecimento para a vigilância pós-comercialização de dispositivos médicos ${ }^{23}$.

Alguns autores reforçam a importância do UDI para aumentar a eficácia e eficiência da vigilância póscomercialização no banco de dados e para a rastreabilidade do DMI, em caso de necessidade de recalls:

\footnotetext{
"Dada a indisponibilidade de um Identificador de Dispositivo (ID), que deve garantir a rastreabilidade do implante em caso de recall do produto, a cooperação com os fabricantes para implementar a Biblioteca RIAP MD integrada com a coleta de dados de registro é crucial. Numa perspectiva futura, o desenvolvimento de um ID como um sistema harmonizado a nível europeu será a melhor forma de garantir a rastreabilidade eficaz dos DMs na União Europeia e apoiar a interoperabilidade de diferentes sistemas para monitorizar a utilização de DMs a nível nacional e internacional nível ${ }^{24}$."
}

O artigo aponta que, na literatura, não existem estudos comparativos entre as legislações relacionadas à UDI. Os autores também apontaram que estabelecer o sistema UDI no ambiente digital é desafiador e que há dificuldades na implementação do sistema de rastreamento baseado em UDI por dois motivos: a legislação pertinente não fornece informações detalhadas sobre como o sistema UDI será implementado, e cada tipo de dispositivo médico tem suas próprias dificuldades de rotulagem UDI. Em particular, na Turquia, os autores observaram que as partes interessadas na indústria de dispositivos médicos, especialmente fabricantes, ainda não estão prontos para o rastreamento baseado em ID e que o sistema de registro atual não é eficaz para rastrear dispositivos médicos e compartilhar dados ${ }^{25}$. 


\section{Conclusão}

O estudo tratou da análise dos 126 artigos recuperados da base de dados Scopus, publicados em 88 periódicos de 2006 a 2018, todos estrangeiros, sem registro de publicações do Brasil ou em português, o que indica a baixa produtividade dos pesquisadores brasileiros na UDI e assuntos de rastreabilidade do DMI.

Ficou demonstrado o aumento do número de publicações ao longo de 12 anos, bem como a origem dos artigos, os periódicos mais publicados sobre o tema e suas localizações geográficas. Quanto aos autores, percebeu-se que, no universo de 96 indivíduos, 62 artigos (49\%) foram produzidos por 13 deles (14\%); sendo que, na média de três autores, o percentual acumulado de publicação correspondeu a $54,8 \%$ da produção, corroborando com a Lei dos Price.

No que diz respeito às palavras, todas em inglês, a Primeira Lei de Zipf poderia ser parcialmente aplicada, uma vez que não havia relação constante entre posto e frequência. Pelas palavras citadas nos artigos recuperados, constatou-se que $64 \%$ delas foram utilizadas apenas uma vez, enquanto outras, que representaram 36\%, foram utilizadas numa variabilidade de duas a 125 vezes, o que revela uma grande dispersão dos termos indexados usado e sugere uma forte heterogeneidade de temas cobertos em artigos sobre UDI.

Por outro lado, o termo "dispositivo médico" foi utilizado em 58 artigos - quase metade do universo analisado. E apesar de ser uma palavra que não basta para designar um tema ou assunto na área da UDI, ela é incluída em algumas situações, provavelmente como forma de facilitar a indexação das pesquisas realizadas. O termo "Identificação Única de Dispositivo" foi utilizado 23 vezes, enquanto a palavra "rastreabilidade" foi citada apenas 12 vezes, o que talvez corrobore na produção científica recente sobre o assunto.

Ainda sobre as palavras, foi feita sua distribuição por esferas. Assim, surgiram aqueles que são realmente relevantes para indexar o tema desta pesquisa - os da segunda esfera. Ressalta-se que as palavras foram aquelas citadas em tópicos relacionados aos órgãos reguladores e ao Fórum Internacional de Reguladores de Produtos para a Saúde (IFRHP), à metodologia aplicada ao UDI por meio da UDI, ambos vinculados à segurança do paciente, também abordados neste estudo.

Aplicando a segunda lei de Zipf e o ponto $T$ de Goffman, foram encontradas palavras relacionadas ao tema, como "vigilância pós-mercado" e "dispositivos médicos", corroborando a Lei de Zipf no que diz respeito à indexação temática, derivada da análise de uma amostra representativa de documentos sobre um determinado assunto. Na região de transição, portanto, estão as palavras com maior conteúdo semântico em um determinado texto, indicando que é possível determinar o conteúdo semântico das publicações com a aplicação das leis de Zipf e suas derivações.

Além disso, destacaram-se os periódicos que realizaram a pesquisa, as instituições às quais estão vinculados e sua localização geográfica. Nesse sentido, fica evidente que o tema permanece isolado entre pesquisadores dos Estados Unidos e países europeus, confirmando a formação de redes de colaboração internacional e interinstitucional, bem como o envolvimento dos países na produção e inserção de novas tecnologias em saúde no Brasil. mercado.

Apesar de ter como base teórica dados e fatos empíricos, a Lei de Lotka e a Lei dos Preços confirmam a hipótese de que os periódicos mais voltados ao tema são os mais produtivos, além de mostrar que quanto mais específico o tema, mais delimitada será a possibilidade de identificação de grupos de elite de autores.

Apesar da notória importância do tema UDI e da rastreabilidade para a área do conhecimento em saúde e segurança do paciente, o engajamento científico nas revistas de maior impacto não tem sido diretamente proporcional. Porém, o fato de ser um tema de ciência e tecnologia na área da saúde justificaria o maior percentual de publicações em um periódico específico da engenharia.

Outro aspecto importante dentro das áreas de conhecimento do domínio de artigos e periódicos recuperados, que precisa ser considerado, refere-se à hegemonia do campo da medicina na publicação de artigos, uma vez que o desenvolvimento de tecnologias em saúde está fortemente vinculado ao seu uso. nesta área. área (testes, desenvolvimento e utilização no mercado), corroborando o surgimento de periódicos relacionados à gestão da informação, gestão em administração, economia e tecnologia da informação.

A título de contribuição, os resultados deste estudo, bem como o seu referencial metodológico, podem servir de base para outras pesquisas, preenchendo assim uma lacuna no âmbito da pesquisa bibliométrica em enfermagem. Reforça-se também que pesquisas sobre a tríade UDI versus rastreabilidade versus DMI devem ser mais exploradas pela enfermagem, tendo em vista a atuação desse profissional nas áreas de gestão em saúde, auditoria em saúde, programas de acreditação, qualidade do atendimento em saúde e segurança do paciente ${ }^{26}$.

Como limitação, cita-se o pequeno número de publicações sobre o assunto e o fato de todas as palavras utilizadas estarem em inglês. além disso, não foram utilizados sinônimos para o descritor utilizado, pois o UDI não possui entretenimento. Pode-se inferir que, devido ao número reduzido, não foi possível comprovar a Lei Bibliométrica de Bradford, o que incentiva e instiga novos estudos sobre UDI. 


\section{Referências}

1. Agência Nacional de Saúde Suplementar (ANSS). Relatório final: grupo de trabalho externo de Órteses, Próteses e Materiais Especiais (GTEOPME) [Internet]. Brasília (DF): ANS; 2016 [acesso em 14 fev 2020]. Disponível em: http://www.ans.gov.br/images/stories/Particitacao_da_sociedade/2016_gt_opme/gt-opme-relatoriointegral.pdf

2. Organização Mundial da Saúde (OMS). Segundo desafio global para a segurança do paciente: cirurgias seguras salvam vidas (orientações para cirurgia segura da OMS) [Internet]. Rio de Janeiro (RJ): OMS; (2009) [acesso em 14 fev 2020]. Disponível em: https://bvsms.saude.gov.br/bvs/publicacoes/seguranca_paciente_cirurgias_seguras_salvam_vidas.pdf

3. Tracol P. Materials vigilance and traceability. Orthop Traumatol Surg Res. 2016;102(1):95-103. DOI: 10.1016/j.otsr.2015.05.013

4. Curfman GD, Redberg RF. Medical devices: balancing regulation and innovation. N Engl J Med. 2011;365(11):975-977. DOI: 10.1056/NEJMp1109094

5. Marucheck A, Greis N, Mena C, Cai L. Product safety and security in the global supply chain: issues, challenges and research opportunities. J. Oper. Man. 2011;29(7):707-720. DOI: 10.1016/j.jom.2011.06.007

6. Agência Nacional de Vigilância Sanitária (Anvisa) Resolução n. 2, de 25 de janeiro de 2010. Dispoõe sobre o gerenciamento de tecnologias em saúde em estabelecimentos de saúde [internet]. Brasília (DF): Anvisa; 2010 [acesso em 23 fev 2020]. Disponível em: https://bvsms.saude.gov.br/bvs/saudelegis/anvisa/2010/res0002_25_01_2010.html

7. Ministério da Saúde (BR). Manual de boas práticas de gestão das Órteses, Próteses e Materiais Especiais (OPME) [Internet]. Brasília (DF): MS; 2016 [acesso em 3 mar 2020]. Disponível em:

https://bvsms.saude.gov.br/bvs/publicacoes/manual_praticas_gestao_proteses_materiais_especiais.pdf

8. International Medical Device Regulators Forum (IMDRF). Medical devices: post-market surveillance: national competent authority report exchange criteria and report form [Internet]. Brasília (DF): IMDRF; 2017 [acesso em 3 mar 2020]. Disponível em: https://www.imdrf.org/sites/default/files/docs/imdrf/final/technical/imdrf-tech-170921-pms-ncar-n14-r2.pdf

9. Lotka AJ. The frequency of distribution of scientific productivity. J. Wash. Acad. Sci [internet]. 1926 [acesso em 3 mar 2020];16(12). Disponível em: https://www.scirp.org/(S(czeh2tfqyw2orz553k1w0r45))/reference/ReferencesPapers.aspx?ReferencelD=2137230

10. Bradford SC. Sources of information on specific subjects. Engineering. 1934;137. DOI: 10.1177/016555158501000407

11. Goffman W. Mathematical approach to the spread of scientific ideas: the history of mast cell research. Nature. 1966;212(5061):449-452. DOI: $10.1038 / 212449 a 0$

12. Goffman W, Newill VA. Generalization of epidemic theory: an application to the transmission of ideas. Nature. 1964;204(4955):225-228. DOI: $10.1038 / 204225 \mathrm{a} 0$

13. Conselho Nacional de Saúde (CNS). Resolução n. 466, de 12 de dezembro de 2012. Considerando o respeito e a dignidade humana e pela especial proteção devido aos participantes das pesquisas científicas envolvendo seres humanos [Internet]. Brasília (DF): CNS; 2012 [acesso em 3 mar 2020]. Disponível em: https://conselho.saude.gov.br/resolucoes/2012/Reso466.pdf

14. Guedes VL, Borschiver S. Bibliometria: uma ferramenta estatística para a gestão da informação e do conhecimento, em sistemas de informação, de comunicação e de avaliação científica e tecnológica. Encontro Nacional de Ciência da Informação [Internet]. 2005 [acesso em 3 mar 2020];6(1). Disponível em: http://www.cinform-anteriores.ufba.br/vi_anais/docs/VaniaLSGuedes.pdf

15. Santos RN, Kobashi NY. Bibliometria, cientometria, infometria: conceitos e aplicações. PBCIB [Internet]. 2009 [acesso em 4 mar 2020];2(1). Disponível em: https://brapci.inf.br/index.php/res/v/119278

16. Price, DJ. Little science, big science. New York: Columbia University Press; 1963.

17. Price DJ. Networks of scientific papers. Science [Internet]. 1965[acecsso em 4 mar 2020];149(3683):510-515. Disponível em: https://jastjournal.springeropen.com/

18. Andrade SR, Schimitt MD, Storck BC, Piccoli T, Ruoff AB. Análise documental nas teses de enfermagem: técnica de coleta de dados e método de pesquisa. Cogitare Enferm. 2018;23(1). DOI: 10.5380/ce.v23i1.53598

19. Booth AD. A "law" of occurrences for words of low frequency. Inf. Control. 1967;10(4):386-393. DOI: 10.1016/S0019-9958(67)90201-X

20. Maisel WH. Semper fidelis: consumer protection for patients with implanted medical devices. N Engl J Med. 2008;358(10):985-987. DOI: 10.1056/NEJMp0800495

21. Altenstetter C. Medical device regulation in the European Union, Japan and the United States: commonalities, differences and challenges. Innovat Eur J Soc Sci Res. 2012;25(4)362-388. DOI: 10.1080/13511610.2012.723328

22. Kramer DB, Tan YT, Sato C, Kesselheim AS. Postmarket surveillance of medical devices: a comparison of strategies in the US, EU, Japan, and China. PLoS Med. 2013;10(9). DOI: 10.1371/journal.pmed.1001519

23. Zippel C, Joschko SB. Post market surveillance in the german medical device sector - current state and future perspectives. Health Policy. 2017;121(8):880-886. DOI: 10.1016/j.healthpol.2017.06.005

24. Torre M, Romaníni E, Zanoli G, Carrani E, Luzi I, Leone L, et al. Monitoring outcome of joint arthroplasty in Italy: implementation of the national registry. Joints. 2017;5(2):70-78. DOI: 10.1055/s-0037-1603899

25. Bayrak T, Çopur FÖ. Evaluation of the unique device identification system and an approach for medical device tracking. HPT. 2017;6(2):234-241. DOI: 10.1016/j.hlpt.2017.04.003

26. Queiroz Freitas dos Santos MA, Moraes dos Santos C, Nogueira Vieira da Silva AL, da Silva Orlandi T, Duarte de Oliveira L. Auditoria de enfermagem: aspectos da qualidade da assistência e do seu registro no prontuário. Glob Acad Nurs. 2020;1(2):e33. DOI: 10.5935/26755602.20200033 